\title{
Taking the Pressure off the Patient - Understanding Digital Rectal Examinations on a Real Subject
}

\author{
Alejandro Granados*, Seth Cox, Naomi Low-Beer, Jenny Higham, Roger Kneebone, and Fernando Bello
}

\begin{abstract}
Better understanding of palpation techniques during unsighted physical examinations has mostly been limited to qualitative and quantitative studies of performance of experts whilst conducting examinations on plastic benchtop models. However, little is known about their performance when conducting such examinations on real subjects. Objective: The aim of this paper is to better understand palpation techniques of experts whilst conducting a Digital Rectal Examination on a real subject. Methods: We recruited four consultants from relevant specialties and asked them to conduct two DREs on a Rectal Teaching Assistant whilst wearing small position and pressure sensors on their examining finger. We segmented the relevant anatomy from an MRI taken of the pelvic region, registered 3D models and analysed retrospectively performance in relation to executed tasks, supination/pronation, palpation convex hull and pressure applied. Results: Primary care consultants examined the anatomy more holistically compared to secondary care experts, the maximum pressure applied across experiments is $3.3 \mathrm{~N}$, overall the pressure applied on the prostate is higher than that applied to rectal walls, and the urologist participant not only applied the highest pressure but also did so with the highest most prominent frequency $(15.4$ and $25.3 \mathrm{~Hz})$. Conclusions: The results of our research allow for better understanding of experts' technical performance from relevant specialities when conducting a DRE, and suggest the range of pressure applied whilst palpating anatomy. Significance: This research will be valuable in improving the design of haptics-based learning tools, as well as in encouraging reflection on palpation styles across different specialities to develop metrics of performance.
\end{abstract}

Index Terms - Tracking technology, pressure sensors, quantitative analysis, visualisation, palpation, imaging, performance, metrics, simulation.

\section{INTRODUCTION}

U nsighted physical examinations, such as Digital Rectal Examinations (DRE) and Bimanual Vaginal Examinations (BVE) require the integration of interpersonal, psychomotor, cognitive and communication skills, clear patient orientation and instruction, appropriate feedback to the patient regarding the findings, and a meticulous, thorough, and skilful technique [1]. These examinations are used to diagnose anorectal [2], prostate and gynaecological abnormalities and are performed by a range of clinicians in primary, secondary and community

Submitted on: March $1^{\text {st }} 2019$. This work was partly supported by the EPSRC, the London Deanery and the Health Education North West London.

A. Granados (correspondence e-mail: alejandro.granados@kcl.ac.uk)was with Imperial College London and now is with King's College London, UK. S. Cox was with Imperial College London. N. Low-Beer was with Imperial College London and now is with Lee Kong Chian School of Medicine, Singapore. J. Higham was with Imperial College London and now is with St. care settings, including general practitioners, continence, urologist and colorectal nurse practitioners, urologists, gynaecologists, and colorectal surgeons.

Traditional learning resources for medical students to train on DRE include examinations texts, diagrams of 2D sagittal views of pelvic anatomy, plastic benchtop models, Rectal Teaching Assistants (RTA) and patients during clinical hospital rotations $[1,3]$. However these examinations are challenging to learn and teach for a number of reasons. Patient anatomical variability is difficult to learn from diagrams, plastic benchtop models are limited in their realism and repertoire of pathologies, and RTAs are mostly healthy volunteers and rarely available in medical schools. Moreover, trainees are randomly exposed to clinical cases amid anxieties due to the nature of an intimate examination whilst practising on patients. More crucially, there is a lack of feedback during and after training as well as an ineffective assessment of performance as a result of being unable to see trainees' performance.

Whilst most approaches aim to provide more efficient mechanisms for training such as virtual haptics-based simulators [4-6] or augmenting their learning experience via see-through views of the anatomy [7], little is known about palpation skills and what constitutes adequate performance. Consequently, due to inexistent validated metrics, medical students are unable to reflect on performance and receive effective feedback from medical tutors. Qualitative approaches in the form of Cognitive Tasks Analysis (CTA) describing the necessary steps to conduct an examination [8,9] and quantitative approaches that use sensors technology to track the position of the examining finger and measure the amount of applied pressure [3,10-12] have been proposed to study performance on benchtop models. Unfortunately, our understanding of palpation skills is limited by how realistic the benchtop models are and is affected by the lack of integration of other skills that are necessary whilst performing intimate physical examinations.
George's Hospital, University of London, UK. R. L. Kneebone is part of the Centre for Engagement and Simulation Science, Chelsea \& Westminster Hospital, Imperial College London, UK. F. Bello is part of the Simulation and Modelling in Medicine and Surgery group, Centre for Engagement and Simulation Science, Chelsea \& Westminster Hospital, Imperial College London, UK. 
Our motivation is to better understand palpation techniques of unsighted examinations by experts on human subjects. The aim of this paper is to provide qualitative and quantitative insights into the tasks performed during DRE on an RTA using sensors technology. Our main contributions are: 1) a fully comprehensive trajectory and pressure analysis of performance during DRE on a human subject, 2) extensions to previous methods originally designed on benchtop models and adapted to human subjects, and 3) a comparison of performance of four relevant clinical specialties within a contextualised clinical setting. To the best of our knowledge, this is the first time a complete analysis of performance of experts on a real subject is presented, apart from a system feasibility study investigating BVE on Gynaecological Teaching Assistants [11].

\section{Materials AND MethodS}

\section{A. Position Tracking and Pressure Sensing}

We use a real-time visualisation and analysis framework $[11,12]$ based on an electromagnetic tracking system (Aurora Northern Digital Inc. Ontario, Canada) using a 6DOF position sensor coil (Aurora Micro $6 \mathrm{DOF} 0.8 \mathrm{mmx} 9 \mathrm{~mm}$ ) and a

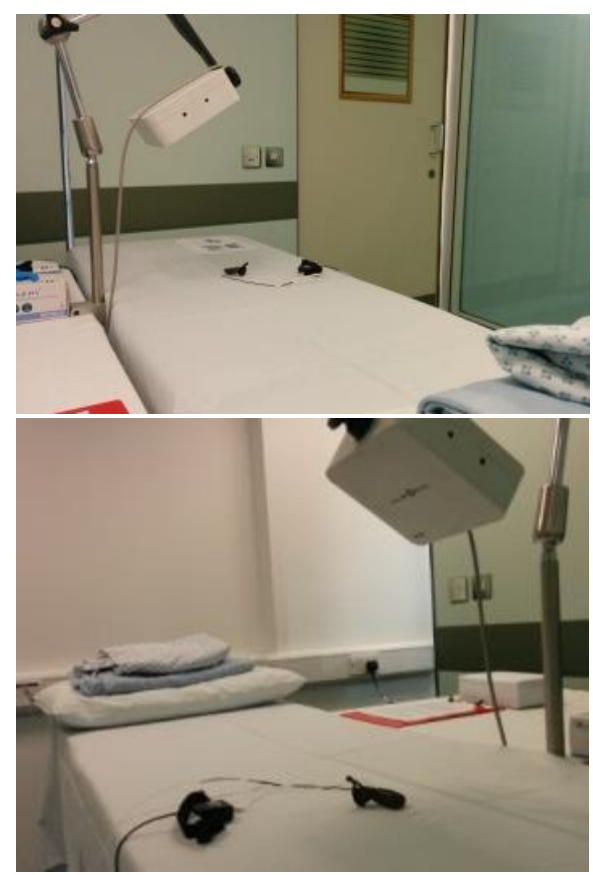

Fig. 1. Simulation environment for our experimental study. During each time slot, we recorded performance of a clinician doing two DREs on a RTA whilst wearing a position and a pressure sensor on the examining finger. The RTA wore a dressing gown and lay in left lateral position under the Aurora electromagnetic tracker.

capacitive pressure sensor system (FingerTPS Pressure Profile System, Inc. USA) with one pressure pad.

\section{B. Experimental Study}

Four clinical experts from four relevant clinical specialties, namely a General Practitioner (GP), a colorectal Nurse Practitioner (NP), a Urologist (UR) and a Colorectal Surgeon (CR) were recruited to perform a DRE on a Rectal Teaching Assistant (RTA), the only one available in the UK at the time of this study (2016). Ethics approval was granted by the NHS
National Patient Safety Agency Research Ethics Committee (reference number: 09/H0701/68). The four participants and the RTA signed consent to participate. During a single day, four different time slots, one per clinical expert, were scheduled and the RTA's pelvic region was MRI-scanned at the MR Therapy Centre at St. Mary's Hospital. The study took place in an office space in the Paterson Centre at St. Mary's hospital, London, UK. We use a Portable Assessment Environment (PAE) system developed by our group consisting of hard panels with images printed on them to contextualise the clinical setting during our study (Fig. 1).

Before entering the examination room, the clinical expert was required to remove any metallic parts from his/her body and to wear both a magnetically-tracked position sensor coil and a pressure sensor pad before wearing clinical gloves. Before examining the RTA, the clinical expert was asked to do a pressure sensor calibration and a finger orientation calibration [12]. Participants were asked to follow a progress bar on a computer screen while calibrating the pressure pad with the reference sensor within the FingerTPS Chameleon ${ }^{\circledR}$ software to guarantee that the calibration was similarly done by all participants. We also minimised drift of pressure sensors by following the manufacturer's recommendations, including: use of correct size of pressure pad, ensure the pad is not loose, allow light use for few minutes before calibration and gently move examining finger to discard any changes in magnitude. We also set the baseline to zero before starting each examination.

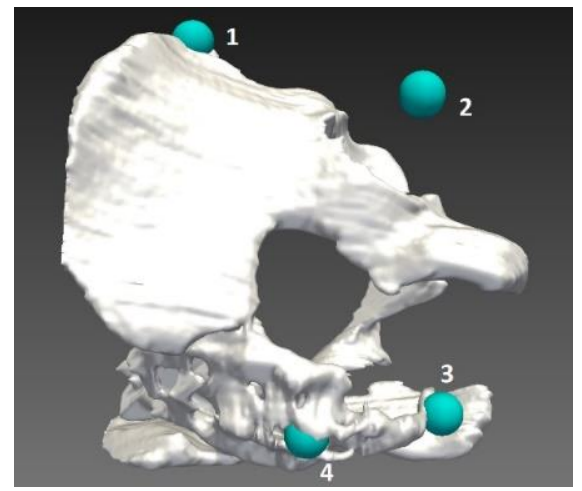

Fig. 2. Bone anatomical landmarks touched on RTA by clinicians before examination: 1) anterior superior iliac spine, 2) greater trochanter, 3) end of coccyx, and 4) sacrum/lumbar spine. Landmarks are used for image registration to position sensors space.

The RTA was then asked to undress and lay on the bed on his left side with his pelvic region just underneath the magnetic tracker. Participants were instructed that movement of the RTA should be avoided once landmarks are recorded and until the two examinations have been fully performed. The clinical expert was asked to touch four different landmarks on the RTA with her/his index finger (tracked by our system) in the following order: 1) anterior superior iliac spine, 2) greater trochanter, 3) end of coccyx, and 4) sacrum/lumbar spine (Fig. 2). These landmarks were used for registration of MRI segmented anatomy with sensors data. Clinical experts were then asked to perform two DREs while position and pressure data was recorded. 


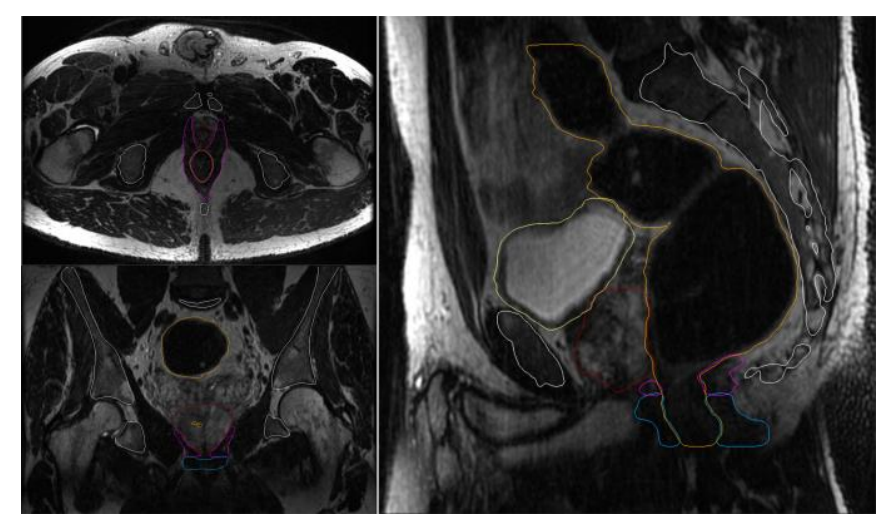

Fig. 3. Axial, coronal and sagittal planes of manual and semi-automatic segmentation.

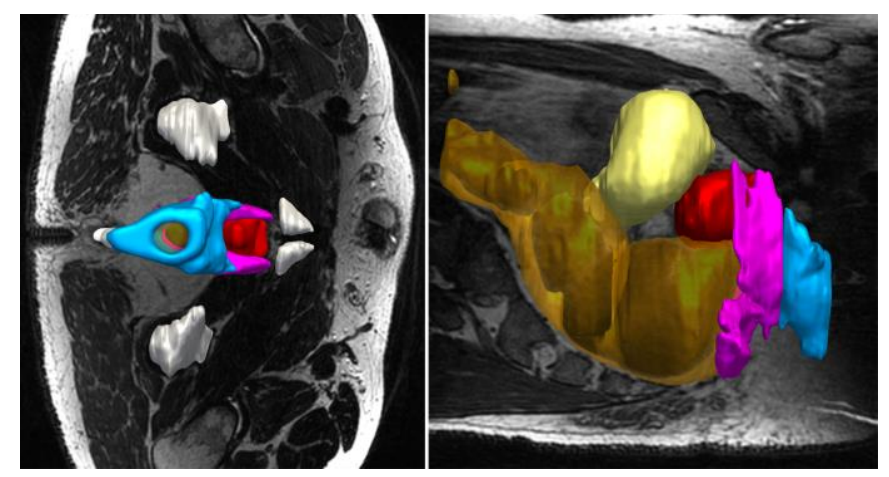

Fig. 4. 3D models of relevant pelvic anatomy including: pelvis (white), anal canal (blue), pelvic floor (pink), rectum (translucent orange), prostate (red) and bladder (yellow).

\section{Image Segmentation}

Delineation of the necessary pelvic anatomy was undertaken under supervision and guidance from a consultant radiologist at Chelsea and Westminster Hospital, London, UK (Fig. 3). We followed $[13,14]$ to correctly identify crucial structures for our analysis, namely the anal canal, puborectalis sling and pelvic floor structures (Fig. 4 left).

Pelvic anatomical structures including the prostate, bladder, rectum, anal canal, a subsection of the pelvic floor and pelvis were manually and semi-automatically segmented using ITKSNAP (http://www.itksnap.org) and post-processed in MeshLab (http://www.meshlab.net) using a Two-Step Smooth and Laplacian Smoothing filters to correct artefacts from the segmentation process (Fig. 4).

\section{Visualisation and Analysis Framework}

The visualisation and analysis framework introduced in $[11,12]$ was adapted and extended for the Unity game engine (https://unity3d.com) to facilitate the analysis with improved graphics rendering (Fig. 5). The segmented organs were manually aligned to the landmarks touched by the clinician during the study and subsequently registered using the Iterative Closest Point (ICP) algorithm.

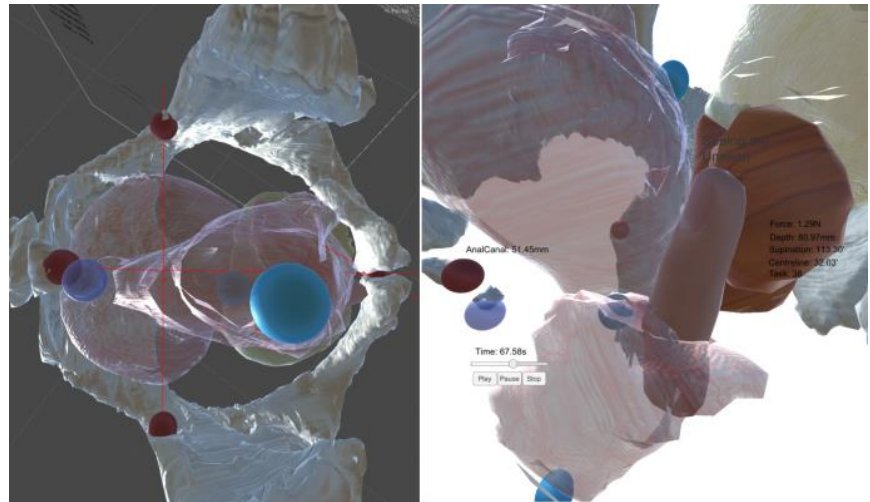

Fig. 5. Visualisation and analysis framework in Unity3D. Two different views of the pelvic anatomy whilst playing back the performance of a consultant examining the RTA. Anal canal and rectum are shown with translucent textures, prostate in red, bladder in yellow and pelvis in white colours. Left: the pelvis is used as a reference frame (red lines) to compute supination/pronation. Right: a centreline is computed between three points: the anus, puborectalis sling and above base of prostate (shown as blue spheres) to compute finger insertion depth.

We performed task analysis by replaying each of the eight examinations and annotating the steps performed together with their observed time intervals based on an adapted version of a Cognitive Task Analysis for DRE [8].

To facilitate analysis, we took four landmark points of the pelvis as a reference frame and built quadrants of the pelvis that are used to compute supination and pronation of the finger (Fig. 5 left). North and south coordinates (at zero and 180 degrees) refer to right and left lateral position, respectively. East and west coordinates refer to anterior (where prostate is located) and posterior (at the level of coccyx/sacrum), respectively. Finger insertion depth is computed based on the position of the tip of the finger projected to a centreline, which is constructed with three anatomical locations: 1) the anus, 2) puborectalis sling located between the anal canal and the rectal ampulla, and 3) a position along the rectum and above the base of the prostate opposite the seminal vesicles. The location of the first two landmarks was confirmed by using a priori knowledge of pressure data, i.e. from gentle pressure applied by clinicians upon initial finger insertion, and pressure recorded at its highest during finger insertion, respectively. Anatomical locations are shown as blue spheres in Fig. 5 right and pressure data is shown in Fig. 8 (see extreme points of red series at the beginning of the examination).

We also extended our analysis of palpation skills by computing a palpation convex hull inside the rectal ampulla to better understand the palpation space explored by clinicians and by investigating prostate examination in the frequency domain. 
TABLE I

OBSERVED TASKS OF FOUR CONSULTANTS DOING TWO DRES ON A RTA

\begin{tabular}{|c|c|c|c|c|c|c|}
\hline Anatomy & Task & Description & GP & NP & UR & CR \\
\hline anus & 24 & apply gentle pressure & $\bullet \circ$ & $\bullet \circ$ & $\bullet \circ$ & $\bullet \circ$ \\
\hline \multirow[t]{3}{*}{ anal canal } & 25 & insert finger into sphincters anteriorly & & & $\bullet \circ$ & \\
\hline & 26 & insert finger into sphincters posteriorly & $\bullet \circ \circ$ & $\bullet \circ$ & & $\bullet \circ$ \\
\hline & 27 & assess sphincter tone & $\circ$ & $\cdot$ & & \\
\hline rectum & 28 & insert finger beyond sphincter into rectum & $\bullet \circ$ & $\bullet \circ$ & $\bullet \circ$ & $\bullet \circ$ \\
\hline $\operatorname{coccyx}$ & 29 & palpate coccyx & $\bullet \circ \circ$ & $\bullet$ & & $\circ$ \\
\hline \multirow[t]{4}{*}{ rectum } & 30 & finger insertion & $\bullet \circ ০ ০$ & & & \\
\hline & 31 & palpate rectal walls & $\bullet$ & $\bullet$ & & \\
\hline & 32 & sweep of rectal walls & $\bullet \bullet \circ \circ$ & $\circ$ & & $\bullet \bullet$ \\
\hline & 33 & compliance on rectal walls & & $\bullet \bullet \circ$ & & \\
\hline \multirow[t]{7}{*}{ prostate } & 34 & sweep / inter-lobe & $\bullet \circ \circ$ & & & \\
\hline & 35 & inter-lobe palpation & & $\bullet \circ$ & $\bullet \circ$ & \\
\hline & 36 & global palpation (base-mid-apex) & $\circ$ & & ০ & \\
\hline & 37 & left lobe palpation & $\bullet \bullet \circ$ & & & \\
\hline & 38 & right lobe palpation & $\bullet \bullet \circ \circ$ & $\bullet \circ$ & $\circ$ & $\bullet \bullet \circ$ \\
\hline & 39 & compliance (pad of finger) & $\bullet$ & $\bullet$ & & \\
\hline & 40 & compliance (top/side of finger) & & $\bullet$ & & \\
\hline rectum & 42 & finger removal & $\bullet \circ$ & $\bullet$ & $\bullet \circ$ & $\bullet \circ$ \\
\hline anal canal & 43 & finger removal & $\bullet$ & $\bullet \circ$ & $\bullet$ & $\bullet$ \\
\hline
\end{tabular}

First examination is shown in black circles and the second one in white circles.

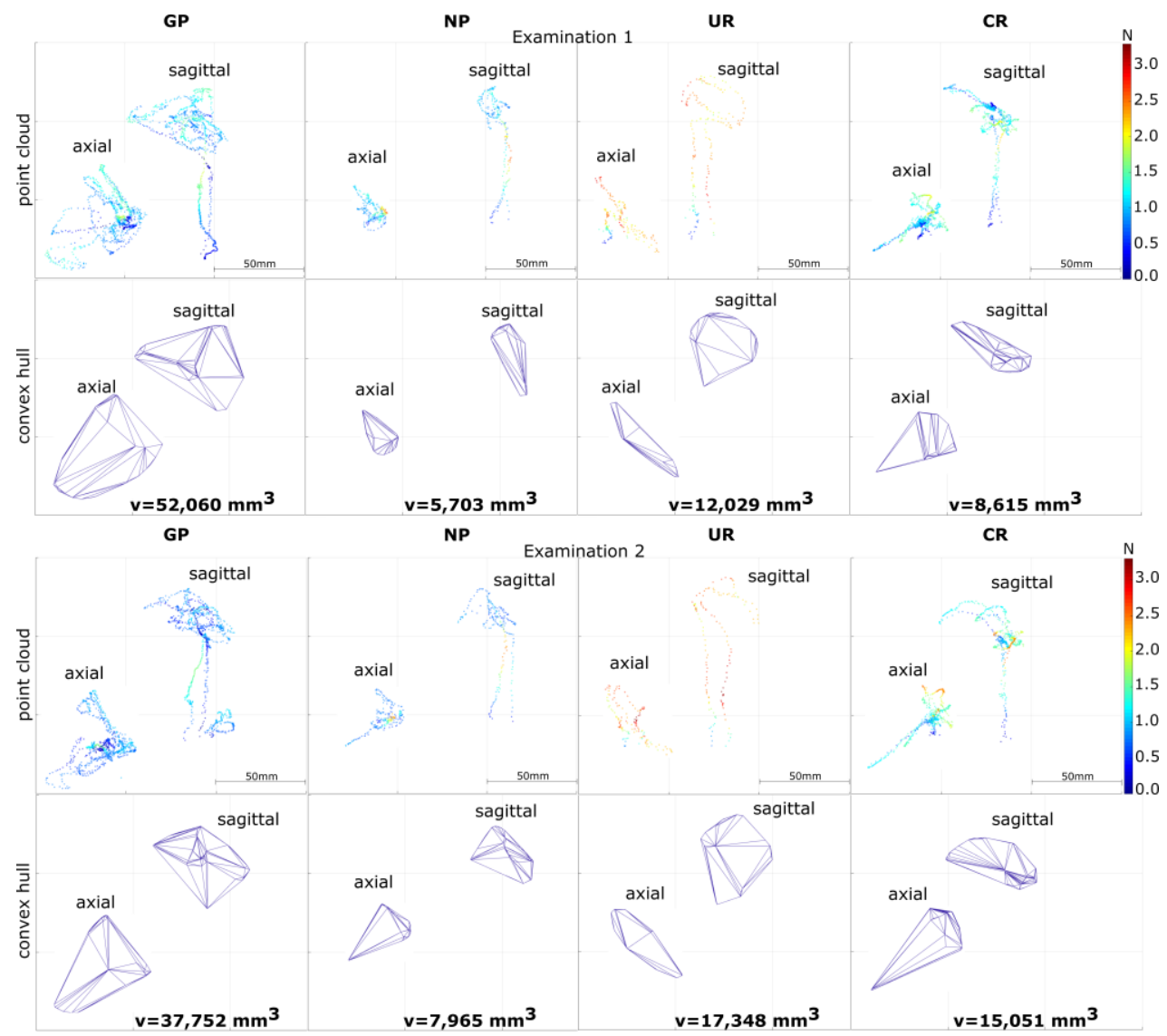

Fig. 6. Position (point cloud) and pressure (colour-mapped) data of two examinations performed by each clinician, together with a convex hull (and its volume) of those points within the rectal walls. 


\section{RESULTS}

\section{A. Task Analysis}

Table 1 shows the results of the conducted task analysis by listing those executed tasks that were observed during playback. Although we started our analysis with a subset of tasks from the original CTA for DRE, we annotated steps based on the palpated anatomy and the type of exploratory movement observed. Therefore, task numbers do not correspond to those ones reported in [8].

\section{B. Palpation Convex Hull}

A convex hull was computed in MATLAB for each examination based on the finger positions within the rectal walls in order to quantify the volume of the palpated internal space. Fig. 6 shows a sagittal and an axial view of the position of the examining finger (point cloud) and its computed convex hull. Each recorded position is colour-mapped with the amount of pressure applied on the anatomy.
We found that the GP palpated the internal anatomy with the largest volume $\left(\mathrm{v}_{1}=52,060 \mathrm{~mm}^{3}\right.$ and $\left.\mathrm{v}_{2}=37,752 \mathrm{~mm}^{3}\right)$, followed by the UR $\left(\mathrm{v}_{1}=12,029 \mathrm{~mm}^{3}\right.$ and $\left.\mathrm{v}_{2}=17,348 \mathrm{~mm}^{3}\right)$, the CR $\left(\mathrm{v}_{1}=8,615 \mathrm{~mm}^{3}\right.$ and $\left.\mathrm{v}_{2}=15,051 \mathrm{~mm}^{3}\right)$ and with the smallest volume palpated by the NP $\left(\mathrm{v}_{1}=5,703 \mathrm{~mm}^{3}\right.$ and $\left.\mathrm{v}_{2}=7,965 \mathrm{~mm}^{3}\right)$. With the exception of the UR, the palpation space explored by clinicians is posterior, whereas the space palpated by the UR appears anteriorly, that is, towards the prostate.

\section{Supination and Pronation}

Based on the quadrants computed using the pelvis as a reference frame, supination and pronation are plotted in polar coordinates of penetration depth and the number of degrees that the examining finger supinated/pronated for each examination (Fig. 7). Similar patterns are observed for both examinations of the same participants and conspicuous differences are evident across participants. For example, the GP and the NP alternate between the rectum and the prostate more frequently, the UR focus only on the prostate and the palpation of the right lobe with the finger facing upwards is evidently done by the CR.

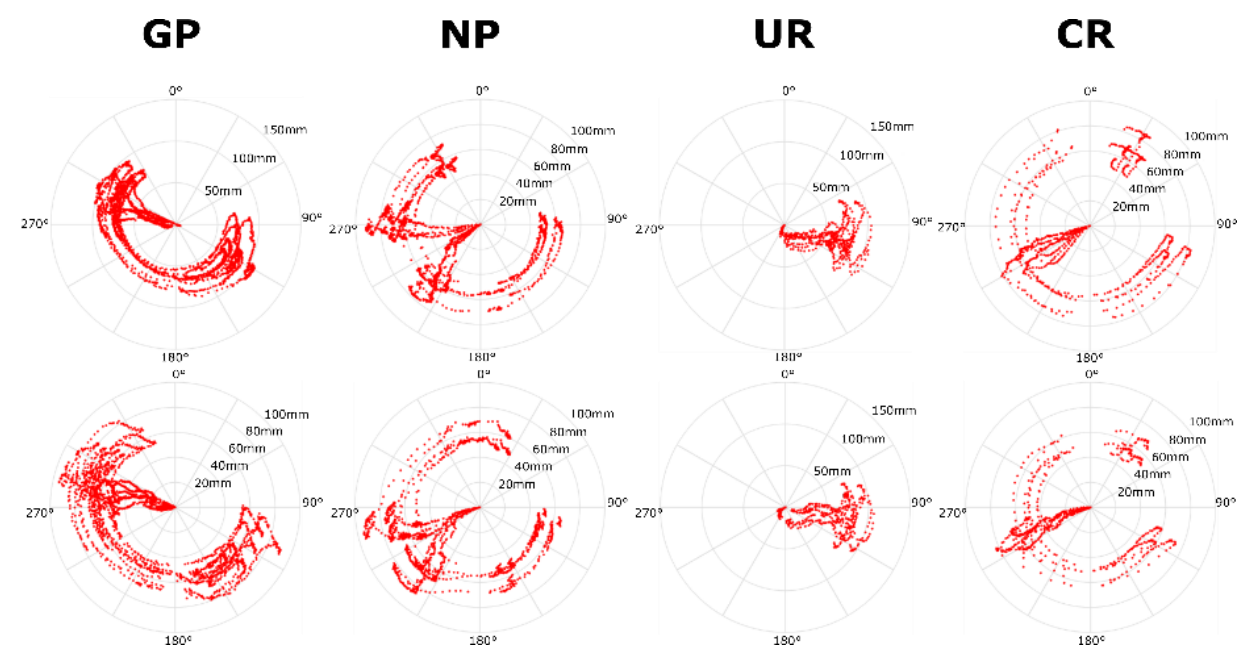

Fig. 7. Palpation styles during both examinations by four consultants of different specialties. First examination is shown above and second below. From left to right: GP, NP, UR and CR.
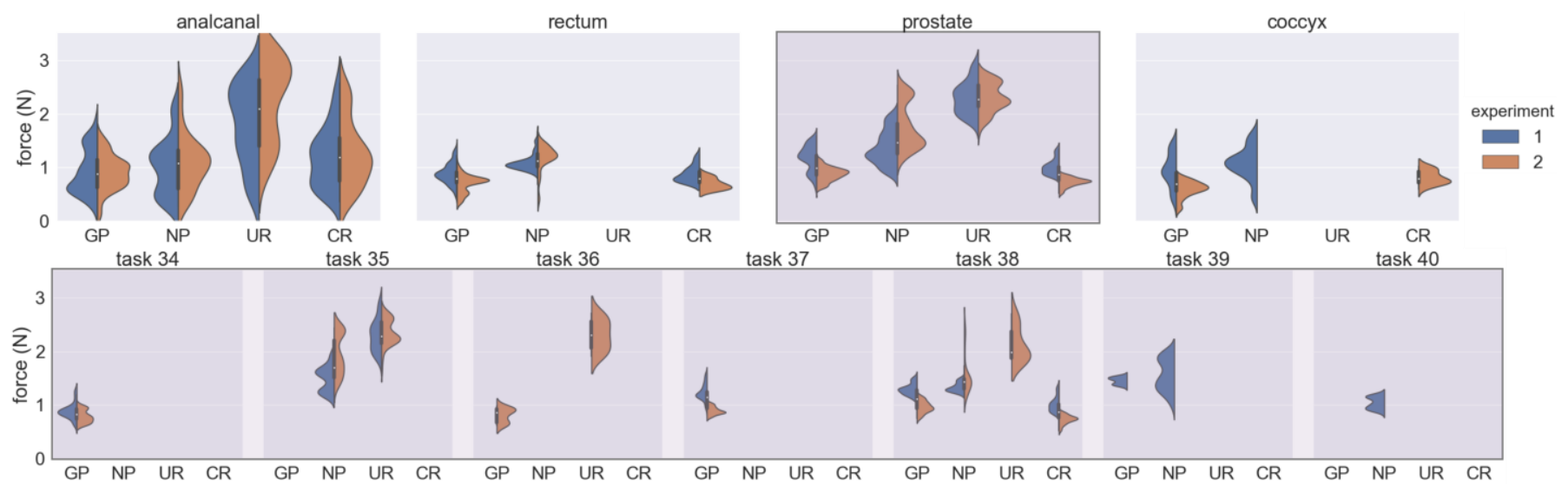

Fig. 9. Violin plots of pressure applied by each participant stratified by anatomy (top) and by tasks related to prostate palpation (bottom). Finger insertion (28) and removal (42) tasks in the rectum are not plotted. 


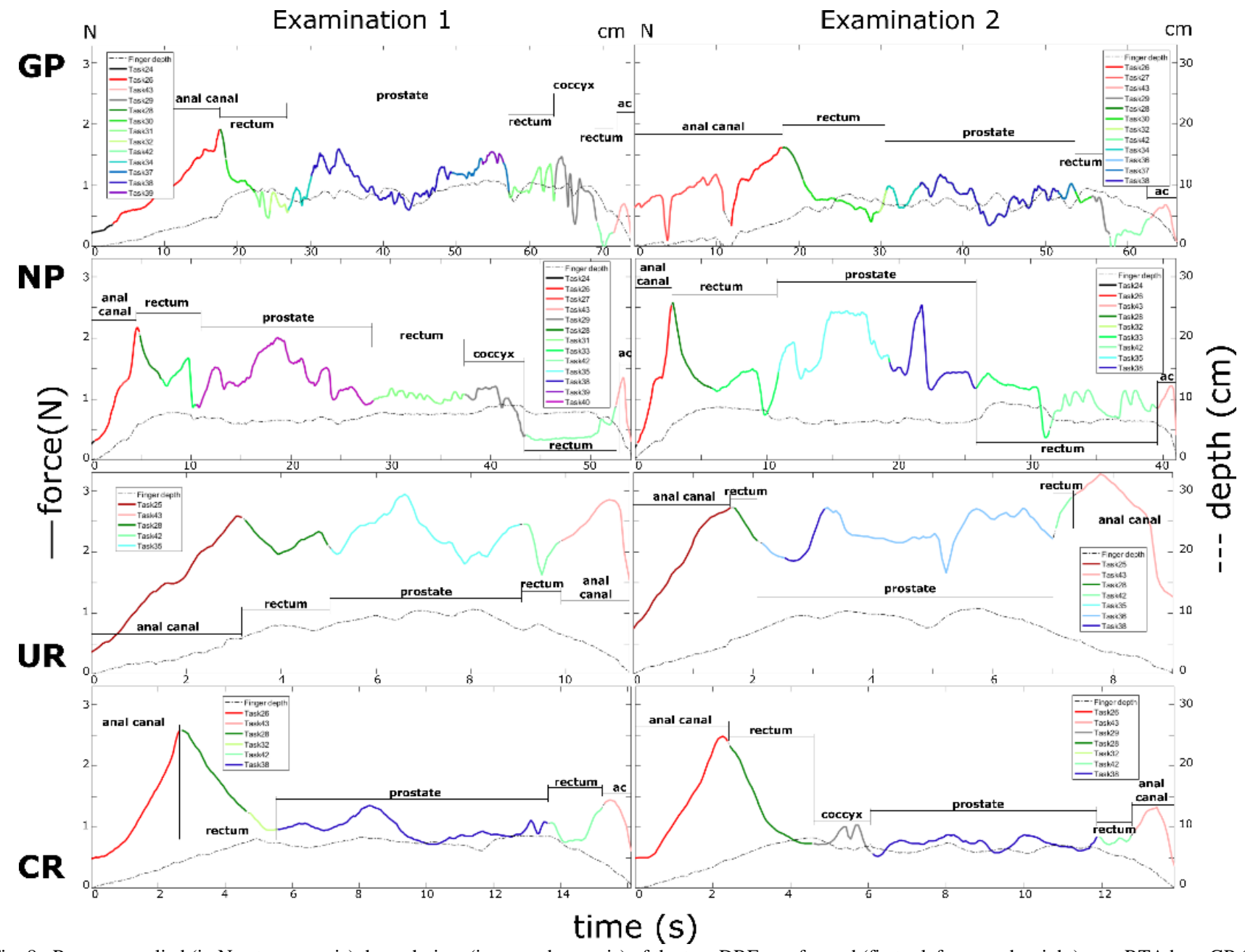

Fig. 8. Pressure applied (in Newtons; y axis) through time (in seconds, $x$-axis) of the two DREs performed (first - left; second - right) on a RTA by a GP (1 $1^{\text {st }}$ row $),$ a NP $\left(2^{\text {nd }}\right.$ row $)$, a UR ( $3^{\text {rd }}$ row $)$ and a CR ( ${ }^{\text {th }}$ row $)$. Annotation of tasks is shown in different colour tones, whilst palpating the anal canal (red), rectum (green), prostate (blue) and coccyx (grey). Finger insertion depth (in $\mathrm{m}$ ) is plotted as a dashed grey line.

\section{Pressure Analysis}

The maximum pressure observed during experiments was $1.9 \mathrm{~N}, 2.6 \mathrm{~N}, 3.3 \mathrm{~N}$ and $2.6 \mathrm{~N}$ by the GP, NP, UR and CR, respectively. The pressure applied during each DRE is shown in Fig. 8. Tasks executed by clinicians are colour-mapped by regions of the anatomy, including: anal canal (red), rectum (green), prostate (blue) and coccyx (grey).

We observed that one of the highest pressure magnitudes observed is a peak during finger insertion beyond the anal sphincters into rectum (Fig. 8). This is explained by the fact that the space inside the anal canal is more constrained compared to that of the rectum. Interestingly, we observed an increasing force rather than a steady force throughout the anal canal once the finger is inserted. The peak magnitude also corresponds visually to a position between the segmented models of the anal canal and the rectum. It can also be noticed that the UR applied even slightly higher pressure on other tasks (including finger removal) compared to the peak pressure applied during finger insertion, and overall applied higher pressure compared to other specialists.
The GP applied a high pressure whilst palpating the right lobe (just after sweeping of rectal walls and inter-lobe) and then during compliance at around $1.5 \mathrm{~N}$. The sweep of rectal walls was done with lower pressure compared to the pressure applied when reaching the prostate and palpating the lobes. The GP also applied slightly less pressure during the second examination. The difference in pressure applied to the rectum and prostate is lower by GP and CR, and larger by the NP (Fig. 9).

Similar to $[10,12]$, pressure data was transformed from the time domain to the frequency domain in MATLAB R2017a to investigate the most prominent frequency and its corresponding power of applied pressure of each examination when palpating the prostate (Fig. 10). We found that the UR palpated not only with the highest pressure but also with the highest most prominent frequency $(15.4 \mathrm{~Hz}$ and $25.3 \mathrm{~Hz})$ compared to other clinicians, with frequencies smaller than $10 \mathrm{~Hz}$. The examinations done by the NP are characterised by the lowest most prominent frequency of palpation $(0 \mathrm{~Hz}$ and $3.7 \mathrm{~Hz})$ and the highest power ( 0.86 and 0.8 ) of normalised pressure. 

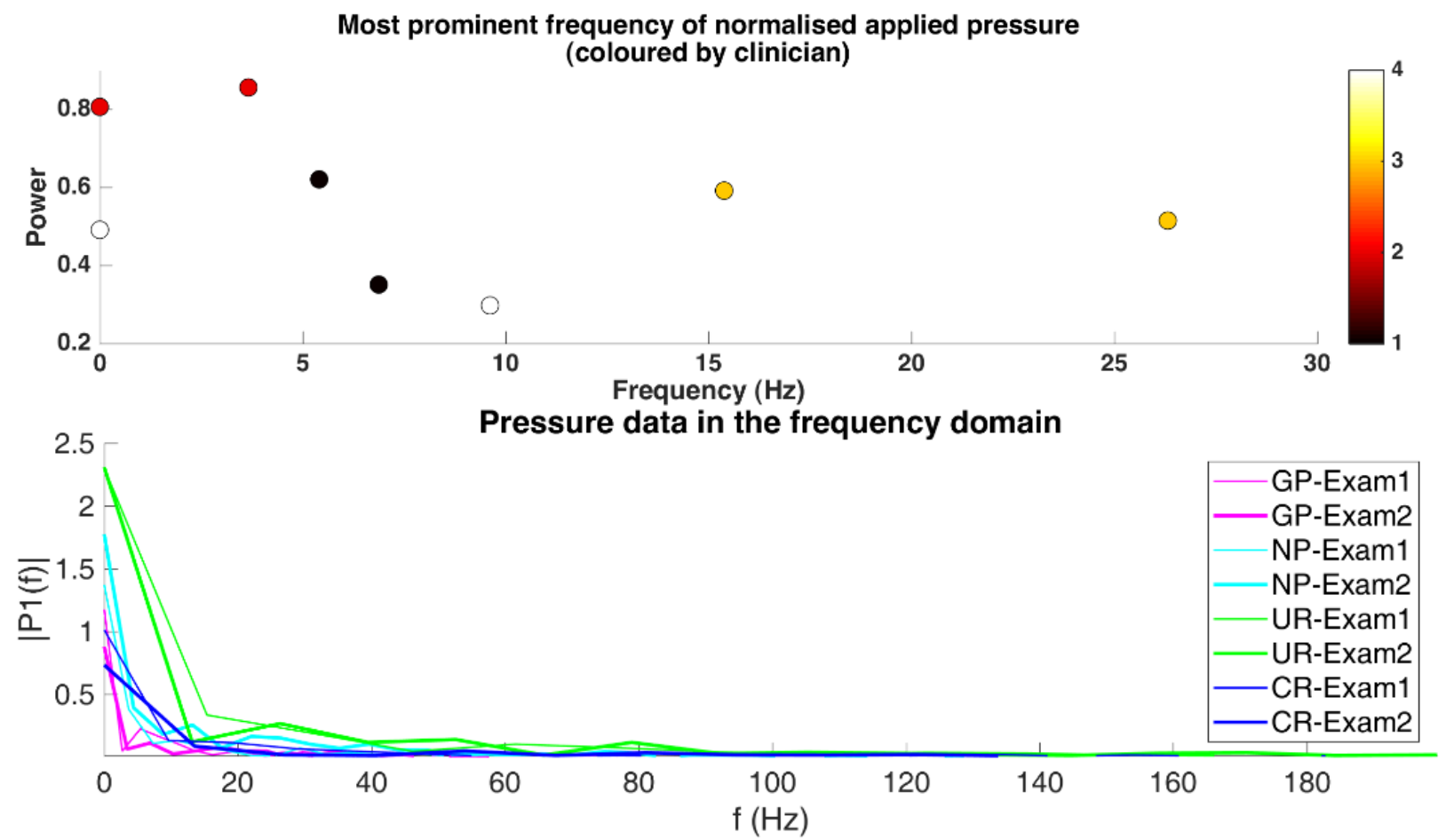

Fig. 10. Pressure analysis in the frequency domain. Left: most prominent frequency of normalised applied pressure coloured by clinician (GP -1 ; NP -2 ; UR - 3; CR - 4). Right: maximum power (pressure applied) during each examination in non-normalised pressure.

\section{DISCUSSION}

Through a visualisation and analysis framework, we were able to record the performance of experts doing two DREs on a real subject whilst wearing position and pressure sensors. Together with MRI-scanned segmented pelvic organs and playing back performance, we report tasks executed by clinicians and study the pressure applied throughout unsighted examinations. Based on our observations of position and pressure information, an extended CTA for DRE analysis that better characterises the tasks performed during internal palpation is presented. More importantly, we quantitatively report pressure applied during DRE, and can accurately describe in Newtons what the typical pressures utilised by experts for DRE are, as well as how much more pressure is applied on the prostate compared to the rectal walls, complementing and confirming previous qualitative studies.

The registration of MRI-scanned segmented organs with the landmarks recorded from participants using the tracking sensor required a series of steps including localising the position of the anus and the position between the anal canal and rectum, together with manual registration, and lastly by running ICP.

In our study, primary care physicians (GP and NP) performed DREs with a higher number of tasks and for longer periods of time compared to secondary care doctors (UR and CR).

Related to pressure applied during palpation, the results reported above suggest a range of forces applied during DRE from zero to a maximum of $3.3 \mathrm{~N}$. This finding is of crucial importance since there have not been studies, to the best of our knowledge, that report the range of forces applied during DRE on real subjects. This finding can also provide more specific requirements for the design of haptic simulators with a range of forces that need to be rendered by a haptic device.

We found the palpation convex hull useful in estimating the extend of the examination within the rectal ampulla. Compared to previous work that use video cameras to compute the working volume during surgical tasks from light-emitting diodes affixed to surgical gloves [19], our proposed palpation convex hull not only gives a better estimate of volume in contrast to a sphere, but also can be used during unsighted examinations on a real subject. Further studies could investigate whether a non-convex hull (e.g. alpha shapes) might better estimate rectal ampulla working space in the context of internal examinations. In the context of our study, whilst GP, NP and $\mathrm{CR}$ did more supination and pronation movements compared to the UR, the palpation convex hull performed by the GP was the highest. This could be explained by the fact that the GP performed more sweeps of rectal walls compared to other participants and that the maximum finger insertion depth was one of the highest above $100 \mathrm{~mm}$. The palpation styles plotted with polar coordinates could be used for summative feedback and for comparisons of performance between medical students and experts from different specialties.

We also observed that participants consistently applied pressure across examinations (intra-examination) and substantial variability across participants (inter-participant), specially on prostate. However obvious this finding might be, since participants are of different clinical specialities, this is the first time pressure data is reported to confirm it. Across participants and experiments, initial finger insertion reached a peak of about $2 \mathrm{~N}$ and the differences between prostate and rectum walls palpation are pronounced in all candidates but the $\mathrm{CR}$, where pressure applied was around $1 \mathrm{~N}$. 
Similar to previous studies investigating the Dominant Intentional Finger Frequency whilst applying firm pressure with varying intensity on a prostate bench-top model (characterised with water balloons) [10], we observe the most prominent frequencies below $30 \mathrm{~Hz}$, a range most sensible to vibrations relevant to rapidly adapting mechanoreceptors at the fingertip. Similarly, our results using normalised pressure (Fig. 10 top) are within the ranges of those reported in [12] related to the most prominent frequencies and power during prostate palpation on a benchtop model. However, with the exception of the UR consultant, the most prominent frequencies of the rest of the consultants were lower than $10 \mathrm{~Hz}$.

There are some limitations to the study presented in this paper. First, the number of participants and RTA (the only one in the UK at the time of this study) recruited is fairly limited and we envisage running further studies with more clinicians and with real subjects with known anorectal or prostate abnormalities. Related also to participants, self-selection bias and a Hawthorne effect may have had an impact on our findings. Second, whilst every effort has been made to minimise the errors introduced by sensors, it is possible that such errors may have inadvertently impacted the results. Although visual inspection of trajectories and pressure data confirmed that there was no movement of the RTA during examinations, a reference tracking sensor could be attached to the RTA to compensate for any possible movement. Third, whilst the RTA was examined in the left lateral position, the MRI sequence from which the anatomical models were delineated from was conducted in the supine position. Whilst there is evidence in the literature that hip flexion alters the anorectal angle [15] as well as the activity and functionality of the pelvic floor [16], there is little to none empirically quantifying the degree or nature of alteration in the left lateral position when compared with the standard supine position. The complexity of this issue is compounded by the fact that merely moving the position of the body from left lateral to supine, even without flexion of the hips, has in itself been found to significantly move some elements of the abdominal and pelvic anatomy $[17,18]$. Although we are unable to quantify the effect this may have had on the fidelity of the models to the anatomy in the relevant position, we mitigated this error by aligning the anatomy with the data captured from position and pressure sensors, particularly during initial finger insertion through the anal canal.

\section{CONCLUSIONS}

In this paper, we have conducted a fully comprehensive analysis of palpation skills during unsighted DRE on a real subject (RTA - Rectal Teaching Assistant). We demonstrate that our framework is sufficiently robust to be used on human subjects and provides qualitative and quantitative analysis of performance. In addition, extensions to previous studies originally designed on benchtop models, with adaptations to human subjects are presented, including: 1) an improved CTA with higher level of granularity, 2) a palpation convex hull for unsighted examinations, 3) detailed segmentations of pelvic anatomy aligned to sensors, 4) an improved playback tool for analysis, and more importantly, 5) a report of actual pressure values in Newtons, rather than normalised values through an improved sensor calibration protocol. In contrast to other systems that embed sensors on fixed locations in plastic models, by using small and unobtrusive sensors, we were able to study tasks executed, palpation styles and pressure applied by four consultants from relevant specialties that routinely perform DRE. To the best of our knowledge, this is the first time, a qualitative and quantitative investigation of palpation skills of experts performing a DRE on a real subject is reported. In this study, we found that the range of forces applied on a healthy subject are within $0-3.3 \mathrm{~N}$. Whilst different palpation styles (tasks performed, palpation convex hull, supination/pronation, pressure applied) are observed across participants, we observed that palpation is consistent between examinations (intraexaminers variability).

Although it is difficult to generalise given the limited number of participants in this study, our framework was able to provide a detailed view of performance with insights into similarities and differences between examinations and across specialties. The preliminary findings of this paper indicate that unsighted physical examinations on human subjects can be comprehensively quantified resulting in a better understanding of palpation techniques. We foresee that the methods and results presented in this work are key for further studies doing statistical analysis of performance on healthy and abnormal cases, correlating correct diagnosis with quantitative and qualitative measures of performance, as well as investigating improved rating scales for assessment.

In future work, we will study the biomechanical behaviour of the pelvic anatomy on palpation and improve our registration techniques to account for deformation of anatomy of a real subject in DRE lateral position with respect to a MRI of a patient in supine position.

\section{ACKNOWLEDGMENT}

This work was partly supported by the EPSRC, the London Deanery, Health Education North West London and the Imperial Health Charity. We are grateful to the MR Therapy Centre at St. Mary's Hospital, particularly to Ms Paula Robinson and Prof Wladyslaw Gedroyc. Ethical approval. All data were evaluated retrospectively. All studies involving human participants were in accordance with the ethical standards of the institutional and/or national research committee and with the 1964 Helsinki declaration and its later amendments or comparable ethical standards. Conflict of interests. The authors declare that they have no conflict of interest. 


\section{REFERENCES}

[1] C. Popadiuk, M. Pottle, V. Curran, "Teaching digital rectal examinations to medical students: an evaluation study of teaching methods," Acad Med: J Assoc American Med Colleges, vol. 77, no. 11, pp. 1140-6. 2002.

[2] J. R. Weber and J. H. Kelley, "Anus, Rectum, and Prostate Assessment," in Health Assessment in Nursing, pp. 475-495.

[3] R. Balkissoon et al., "Lost in translation: unfolding medical students' misconceptions of how to perform a clinical digital rectal examination," American J Surgery, vol. 197, no. 4, pp. 525-532. 2009.

[4] G. Burdea et al., "Virtual Reality-Based Training for the Diagnosis of Prostate Cancer," IEEE Trans on Biomed Eng, vol. 46, no. 10, pp. 125360. 1999.

[5] Y. Kuroda et al., "Interaction model between elastic objects for haptic feedback considering collisions of soft tissue," Comp Meth Prog in Biomed, vol. 80, pp. 216-24. 2005.

[6] A. Granados et al., "Haptics Modelling for Digital Rectal Examinations," Int Symp on Biomed Sim (ISBMS), pp. 40-49. 2014.

[7] A. Granados et al., "See-through Visualisation for Training and Assessing Unsighted Physical Examinations," Workshop Vir Reality Int and Phys Sim (VRIPHYS), Eurographics, 2017.

[8] N. Low-Beer et al., "Hidden practice revealed: using task analysis and novel simulator design to evaluate the teaching of digital rectal examination," American J Surg, vol. 201, no. 1, pp. 46-53. 2011.

[9] C. Gale, "Can a CTA be developed that is likely to be useful for teaching vaginal examination?," MEd in Surg Edu Thesis. Imperial College London, 2012.

[10] N. Wang et al., "Using a prostate exam simulator to decipher palpation techniques that facilitate the detection of abnormalities near clinical limits," Sim in healthcare: J of the Soc for Sim in Healthcare, vol. 5, no. 3, pp. 152-60. 2010.

[11] A. Granados et al., "Real-Time Visualisation and Analysis of Internal Examinations - Seeing the Unseen," MICCAI, vol. 8673, pp. 617-25. 2014.

[12] A. Granados et al., "Real-time Visualisation and Analysis of Clinicians' Performance during Palpation in Physical Examinations," IEEE Trans on Biomed Eng, vol. 65, no. 9, pp. 2042-51. 2018.

[13] “Gray's Atlas of Anatomy," Churchill Livingstone, 2008.

[14] P. A. Georgiou et al., "Diagnostic accuracy and value of magnetic resonance imaging (MRI) in planning exenterative pelvic surgery for advanced colorectal cancer," Eur J Cancer, vol. 49, no. 1, pp. 72-81. 2013.

[15] R. Sakakibara et al., "Influence of Body Position on Defecation in Humans," Lower Urinary Tract Symp, vol. 2, pp. 16-21. 2010.

[16] T. Halski et al., "Relationship between lower limb position and pelvic floor muscle surface electromyography activity in menopausal women: a prospective observational study," Clinic Interv in Aging, vol. 12, pp. 7583. 2017.

[17] A. R. Deukmedjian et al., "Movement of abdominal structures on magnetic resonance imaging during positioning changes related to lateral lumbar spine surgery: a morphometric study," J Neurosurgery Spine, vol. 16, no. 6, pp. 615-23. 2012.

[18] S. Selçuk et al., "Left lateral position and common gynecologic examining position in anal manometry measurements for evaluation of urogynecologic patients," J Turk Ger Gynecol Assoc, vol. 15, pp. 243-4. 2014.

[19] A-L. D. D'Angelo et al., "Working volume: validity evidence for a motion-based metric of surgical efficiency" The American Journal of Surgery, vol. 211, pp 445-450. 2016.

[20] K. Fisher. "Introduction to Alpha Shapes". Stanford course handouts. 2000. 